\title{
Consensus for physiotherapy for shoulder pain
}

Ingrid Hultenheim Klintberg, Ann M J Cools, Theresa Holmgren, Ann-Christine Gunnarsson Holzhausen, Kajsa Johansson, Annelies G Maenhout, Jane S Moser, Valentina Spunton and Karen Ginn

\section{Linköping University Post Print}

\section{Tweet}

N.B.: When citing this work, cite the original article.

The original publication is available at www.springerlink.com:

Ingrid Hultenheim Klintberg, Ann M J Cools, Theresa Holmgren, Ann-Christine Gunnarsson Holzhausen, Kajsa Johansson, Annelies G Maenhout, Jane S Moser, Valentina Spunton and Karen Ginn, Consensus for physiotherapy for shoulder pain, 2015, International Orthopaedics, (39), 4, 715-720.

http://dx.doi.org/10.1007/s00264-014-2639-9

Copyright: Springer Verlag (Germany) http://www.springerlink.com/?MUD=MP

Postprint available at: Linköping University Electronic Press http://urn.kb.se/resolve?urn=urn:nbn:se:liu:diva-115925 
Consensus for physiotherapy for shoulder pain

\section{Consensus for physiotherapy for shoulder pain}

\author{
Running title: Consensus for physiotherapy for shoulder pain \\ Ingrid Hultenheim Klintberg, PhD, PT Inst. Neuroscience and Physiology/Physiotherapy, \\ University of Gothenburg and Physiotherapy Department, Sahlgrenska University Hospital, \\ Gothenburg, Sweden
}

2

3

4

5

6

7

8

9

10

11

12

13 Theresa M Holmgren, PhD, PT, Div. of Physiotherapy, Department of Medical and Health

14 Sciences, Linköping university, Linköping, Sweden

15

16

17

18

19

20

21

22

23

Ann MJ Cools, PhD, PT, Dept of Rehabilitation Sciences \& Physiotherapy

Faculty of Medicine and Health Sciences, Ghent University

(1)


24 Faculty of Medicine and Health Sciences, Ghent University

25

26 Jane S Moser, MSc, Nuffield Department of Orthopaedics, Rheumatology and Musculoskeletal

27 Sciences, Oxford University Hospitals Trust, United Kingdom

28

29 Valentina Spunton, MSc, University of Genoa, Italy

30

31 Karen Ginn, PhD, PT Discipline of Biomedical Science, School of Medical Sciences, Sydney

32 Medical School, The University of Sydney, Australia

33

34 Corresponding author:

35 Ingrid Hultenheim Klintberg, PhD, PT

36 Mailing address:

37 Department of Physiotherapy

38 Sahlgrenska University Hospital/Mölndal

39 SE 43180 Mölndal

40 Sweden

41 e-mail: ingrid.hultenheim-klintberg@vgregion.se

42 alternative e-mail: ingrid.hultenheim@gu.se

43

44 Disclaimer: none

45

46

47 


\section{$48 \quad$ Abstract}

49 Purpose: Shoulder pain is a common disorder. Despite growing evidence of the importance of

50 physiotherapy, in particular active exercise therapy, little data is available to guide treatment.

51 The aim of this project was to contribute to the development of an internationally accepted

52 assessment and treatment algorithm for patients with shoulder pain.

53 Methods: Nine physiotherapists with expertise in the treatment of shoulder dysfunction met in

54 Sweden 2012 to begin the process of developing a treatment algorithm. A questionnaire was

55 completed prior to the meeting to guide discussions. Virtual conferences were thereafter the

56 platform to reach consensus.

57 Results: Consensus was achieved on a clinical reasoning algorithm to guide the assessment and

58 treatment for patients presenting with local shoulder pain, without significant passive range of

59 motion deficits and no symptoms or signs of instability. The algorithm emphasises that

60 physiotherapy treatment decisions should be based on physical assessment findings and not

61 structural pathology; that active exercises should be the primary treatment approach; and that

62 regular re-assessment is performed to ensure that all clinical features contributing to the

63 presenting shoulder pain are addressed. Consensus was also achieved on a set of guiding

64 principles for implementing exercise therapy for shoulder pain namely: a limited number of

65 exercises, performed with appropriate scapulohumeral co-ordination and humeral head

66 alignment, in a graduated manner without provoking the presenting shoulder pain.

67 Conclusion: The assessment and treatment algorithm presented could contribute to a more

68 formal, extensive process aimed at achieving international agreement on an algorithm to guide

69 physiotherapy treatment for shoulder pain. 
Consensus for physiotherapy for shoulder pain

70 Key words: shoulder pain; physiotherapy; exercise therapy; treatment algorithm; clinical

71 reasoning

72

73

74

75

76

77

78

79

80

81

82

83

84

85 
86

87 Shoulder pain is the third most common musculoskeletal condition presenting to physicians or

\section{Introduction}

physiotherapists within primary healthcare. The prevalence of shoulder pain in the general population has been reported to vary between 7 - 30\% [1-3], it increases with age [2] and has been reported to be higher in women than in in men [3].

Current classification systems for shoulder pain have been shown to be unreliable [4-7] resulting in a lack of diagnostic consistency in relation to shoulder pain. Subacromial pain syndrome is the most common diagnosis for patients presenting with shoulder pain $[8,9]$ and includes rotator cuff syndrome (including rotator cuff tears) tendonitis and bursitis[10]. This painful, disabling condition places a significant burden on healthcare resources[10].

Although surgery followed by post-operative physiotherapy (PT) is utilized in the treatment of shoulder pain, there is growing evidence that surgical intervention does not result in superior patient outcomes compared to PT alone. Physiotherapy has been shown to result in as positive short and long term clinical outcomes as subacromial decompression/acromioplasty [11-15] and acromioplasty plus rotator cuff repair [14] in patients with subacromial pain. In addition, evidence is growing that treating shoulder pain with PT greatly reduces the number of patients undergoing surgery for subacromial pain syndrome $[16,17]$ or rotator cuff tear $[18,19]$.

The main PT intervention for treating shoulder pain and dysfunction is active exercise therapy. Limited available data suggests that implementing a program of physiotherapist supervised exercises confers clinical benefit in the short and longer term when compared to no treatment $[11,12,20]$ or placebo treatment $[11,12]$. A number of reviews have concluded that there is 
107 moderate evidence that active exercises reduce pain and restore function in patients with

108 subacromial pain syndrome $[8,10,21]$.

109 Despite growing evidence of the importance of PT, in particular active exercise therapy, in the

110 treatment of shoulder pain there is no consensus as to the most effective exercise strategy. Many

111 exercises have been proposed and little data is available to guide the physiotherapist in selecting

112 the most appropriate care pathway. The aim of this project was to contribute to the development

113 of an internationally accepted algorithm to guide PT assessment and treatment for patients with

114 shoulder pain and dysfunction.

115

116

117

118

119

120

121

122

123

124

125 
126

127

128

129

130

131

132

133

134

135

136

137

138

139

140

141

142

143

144

145

146

\section{Methods}

In October 2012 seven physiotherapists were invited to attend a two day face-to-face meeting in Sweden, by two of the authors (IHK \& KG) to discuss PT treatment of shoulder pain. The physiotherapists were invited on the basis of their special expertise in the treatment of shoulder dysfunction and/or research into the treatment of shoulder dysfunction published in peerreviewed journals or presented at scientific meetings, as well as residing in Europe at the time of the meeting. The aims of the meeting were to:

1) examine the clinical reasoning that underpins how experienced physiotherapists treat shoulder dysfunction

2) develop a treatment algorithm to serve as a guide for less experienced physiotherapists and to contribute to the process of determining international standard best practice in the PT treatment of shoulder dysfunction.

The meeting was financed by funds raised from a conference organized prior to the meeting at which the majority of meeting participants contributed as speakers.

As a basis for discussions at the face-to-face meeting, the nine participating physiotherapists completed a questionnaire prior to the meeting. The questionnaire related to the following clinical scenario: a patient presenting with shoulder pain of insidious onset with no past history of shoulder dysfunction, interfering with everyday life activities and with evidence of partial or a small full thickness rotator cuff tear by MRI. Participants were asked to state their:

i) priorities/focus of initial PT assessment

ii) short/medium term aims of PT treatment 
Consensus for physiotherapy for shoulder pain

147

iii) principle/s guiding an exercise program including how to progress (increase the difficulty) of exercises

iv) frequency of patient attendance for PT treatment

v) criteria to assess PT treatment effectiveness

vi) duration of PT treatment

Responses to the questionnaire that demonstrated consistency between participants were summarized by IHK \& KG prior to the face-to-face meeting and guided discussion at this meeting to clarify points of agreement and disagreement regarding the clinical reasoning underpinning the PT treatment of shoulder pain. Following two days of face-to-face discussion, including demonstration and explanation of the rationale for the use of various exercise strategies, each participant was asked to prepare an algorithm for the treatment of shoulder pain based on the results of the meeting for future discussion over the internet. Multiple virtual meetings chaired by IHK were held in order to reach consensus on an assessment and treatment algorithm for a patient with shoulder pain. Based on the results of the discussions at each meeting documents were revised and distributed. Participants were required to review these revised documents and return comments/suggestions to IHK who collated and distributed responses in preparation for the next meeting. 
168

169

170

171

172

173

174

175

176

177

178

179

180

181

182

183

184

185

186

187

188

189

\section{Results}

The following consistent responses were reported on the questionnaire distributed to participants prior to the face-to-face meeting:

- priorities and focus during the initial assessment: to evaluate shoulder strength deficits and quality of active movements

- $\quad$ short/medium term PT treatment aims should be achieved primarily by active exercises

- guiding principles for an exercise program, including how to progress the exercises i.e. increase difficulty: good quality shoulder movement; minimal pain increase during exercises; progress from basic (simple) to more functional (complex) shoulder movements

- frequency of attendance and duration of PT treatment: average once per week for a period of three months

No consistency was revealed regarding criteria to assess PT treatment effectiveness.

Discussions at the face-to-face meeting focused on four main issues: the definition of "good quality shoulder movement"; the nature of the pain that was to be avoided or minimised during therapeutic exercise performance; criteria to assess PT treatment effectiveness; and specific exercises to use in the PT treatment of shoulder pain.

Participants agreed on the following description of what constitutes "good quality shoulder movement": co-ordinated (smooth) scapulohumeral movement based on movement analysis research and side-to-side comparison, with correct humeral head positioning in the glenoid fossa and no abnormal compensatory trunk movement. 
Participants also agreed that the pain to be avoided or minimized during therapeutic exercise for

191 shoulder pain is pain located over the shoulder joint and/or upper arm which caused the patient to

192 seek treatment. Other sensations that the patient might describe as uncomfortable, muscle

193 soreness or fatigue were acceptable. Further discussions regarding how much pain should be

194 tolerated during therapeutic shoulder exercise revealed two firmly held positions. Some participants argued that shoulder exercises should be chosen and performed so as not to

196 reproduce the pain for which the patient had sought treatment. The rationale for this view 197 included that pain: a) may indicate that the exercise is too difficult, is not being performed 198 correctly or is not the optimal exercise for the patient; b) may be an indication of overload of 199 stressed tissue; c) may inhibit motor relearning; or d) may reduce a patient's motivation to 200 adhere to the exercise therapy. Other participants argued that some localized pain (VAS $\leq 4 / 10)$ 201 during the performance of therapeutic shoulder exercises which was short lasting: a) may be 202 beneficial to promote tendon healing; b) may guide how to load the tendon; c) and may motivate 203 some patients to adhere to the exercise therapy. All participants did agree that it was important 204 to empower the patient to adhere to an exercise program and to guide the patient to avoid 205 activities or exercises that aggravate the shoulder pain.

206 Extensive face-to-face discussion did not result in participants being able to agree on a battery of 207 tests to assess PT treatment progress and success or a recommended set of exercises to treat 208 shoulder pain.

209 Following 18 months of virtual discussion consensus was achieved on a physiotherapy 210 assessment and treatment algorithm for a patient with shoulder pain. A flowchart illustrating this 211 algorithm is presented as Figure 1. The flowchart summarizes the clinical reasoning process 212 underpinning the different possible pathways of PT assessment and intervention. The following 
notes were developed to accompany the flowchart in order to explain the principles and

214 procedures to optimize the clinical outcome for a patient with shoulder pain.

215 Introduction to the PT assessment and treatment algorithm for a patient with shoulder 216 pain

217 This algorithm is designed to guide PT assessment and treatment for a patient presenting with the 218 following clinical scenario:

- a primary presenting symptom of shoulder pain during activity with minimal pain at rest

- no significant shoulder passive range of motion deficits taking into account the age of the patient

- no symptoms of shoulder instability, i.e. no history of apprehension or apprehension

Guide to using the algorithm

- Treatment aims are to be achieved by an approach in which active exercises are the primary tool. Along with active exercises the clinician might choose additional therapeutic interventions such as passive mobilization, depending on the clinical signs.

- The selection of exercises and treatment modalities should be based primarily on the findings of the clinical assessment and not the structural pathology.

- The clinical assessment is based on an "if this - do that" approach. The yes/no boxes refer to the "weight" of the functional deficit detected e.g. if no or little muscle performance deficit is detected then the clinician should follow the "no" route i.e. will continue the 
assessment in order to determine if a significant reduced cervical and thoracic spine mobility impairment is present. On the other hand if considerable functional muscle deficit is present the clinician should follow the "yes" route i.e. active exercises to address the muscle deficit.

- Clinical re-assessments should be performed regularly: 1) to determine if the prescribed treatment is addressing the major clinical deficits detected; and 2) to address concurrent clinical deficits. For example, if functional muscle deficits resolve following the active exercises prescribed then the yes/no response following re-assessment of muscle performance deficit would be "no". The algorithm should then be explored through the 'no' route to ensure that all clinical features contributing to the presenting shoulder pain are adequately addressed.

- Definite improvements in symptoms (pain, function, muscle performance and/or range of movement) would be expected within 12 weeks. Ongoing improvements may occur after this time.

\section{General principles for prescribing exercises}

- Exercises should not provoke the pain with which the patient presented.

- Some mild to moderate pain $(\leq 4 / 10$ on VAS) due to the effort of doing the exercise can be tolerated but must have subsided within 12 hours.

- The quality of the performance of exercises is crucial and multimodal feedback (e.g. visual, biofeedback, taping) can be used to achieve this. Exercises should be performed with optimal scapular positioning and control without abnormal compensatory trunk movement. 
- Start with:

$\circ$ unloaded proceeding to loaded exercises

- simple exercises e.g. targeting one force couple at the time, proceeding to more complex movements involving multiple force couples.

- slow proceeding to faster exercises

- exercises performed in a conscious manner and progress, by gradually decreasing feedback, to more subconscious / automatic exercise performance

- Dose and progressions relate to the goal of each exercise and should be adjusted in relation to the individual patient. 


\section{Discussion}

275 After extensive discussion and debate the experienced shoulder physiotherapists associated with

276 this project were able to reach agreement on a clinical reasoning algorithm to guide the

277 assessment and treatment for patients presenting with local shoulder pain, without significant

278 passive range of motion deficits and no symptoms or signs of instability. It was decided not to

279 include "evidence of partial/small full thickness rotator cuff tear by MRI", which had been

280 included in the patient description distributed with the initial questionnaire to participants, as part

281 of this clinical scenario as all participants strongly agreed that PT treatment decisions should

282 primarily be based on physical assessment findings. A clinical description was used to describe

283 the target patient population because of the lack uniformity and reliability of the current

284 diagnostic classification system for shoulder pain [4-7]. The algorithm emphasises that

285 physiotherapy treatment decisions should be based on shoulder physical assessment findings and

286 not structural pathology, and that active exercises should be the primary treatment approach. The

287 algorithm also emphasises the need for regular re-assessment to ensure that all the clinical

288 features contributing to the presenting shoulder pain are addressed.

289 The consensus position to base physiotherapy treatment decisions on physical assessment

290 findings and not structural pathology is supported by research relating shoulder symptoms and

291 functional deficits to the presence and extent of structural deficits at the shoulder. Multiple

292 imaging studies have demonstrated the presence of structural tissue failure at the shoulder in

293 people without symptoms [22-24] and a poor relationship has been demonstrated between the

294 level of shoulder pain and disability and the degree of structural deficit detected with imaging

$295[25,26]$. These findings challenge the validity of imaging procedures to identify the source of 
shoulder symptoms and emphasises the importance of a thorough physical assessment as the

297 basis for determining treatment goals.

298 During the face-to face meeting, discussions revealed low agreement between participants 299 regarding the rationale for choosing particular exercises to treat shoulder pain. This resulted in 300 the group being unable to reach consensus agreement on a set of specific exercises to incorporate 301 into a treatment program. However, consensus was achieved on a set of guiding principles for 302 implementing exercise therapy for shoulder pain and dysfunction. In summary, such a program 303 should be individually prescribed for each patient and should consist of a limited number of 304 exercises, performed with appropriate scapulohumeral co-ordination and humeral head 305 alignment, in a graduated manner without provoking the presenting shoulder pain. The 306 consensus position to only prescribe a small number of exercises is supported by research that 307 indicates that lack of time affects exercise adherence negatively [27, 28].

308 One of the aims of this project was to develop a physiotherapy treatment algorithm to serve as a 309 guide to aid less experienced physiotherapists in the treatment of shoulder pain. Although the 310 clinical reasoning algorithm presented achieved consensus agreement from a group of 311 physiotherapists from various world regions it remains to be seen if it proves helpful for less 312 experienced therapists. A future objective is to field test this algorithm to assess its utility to aid 313 the less experienced clinician to achieve optimal clinical outcomes for patients with shoulder 314 pain.

315 The process to achieve consensus in this project was protracted. Although this is to be expected 316 of a process designed to integrate the judgments of "experts", the inevitable communication 317 problems resulting from the lack of uniformity and reliability of the current classification system 
318 for shoulder disorders significantly contributed to the length of the process. A significant portion 319 of both face-to-face and virtual meetings was spent clarifying to which shoulder pain patients the 320 algorithm applied. Future research to develop international best practice guidelines should ensure 321 that this issue is not an impediment to efficient progress.

322 This project employed an informal consultation process between a limited number of

323 physiotherapists with expertise in the treatment of shoulder dysfunction from Europe and

324 Australia to achieve consensus. To further the aim of determining international best practice

325 guidelines in the treatment of shoulder dysfunction a more structured process involving a more 326 representative sample of physiotherapists with an international reputation for expertise in the 327 treatment of shoulder dysfunction is required. The assessment and treatment algorithm presented 328 in this report could be used as an initial trigger document to begin a more formal extensive 329 consultation process to achieve this end. 


\section{Reference}

340 1. Luime JJ, Koes BW, Hendriksen IJ, et al. (2004) Prevalence and incidence of shoulder

341 pain in the general population; a systematic review. Scandinavian journal of

342 rheumatology 33:73-81.

343 2. Linsell L, Dawson J, Zondervan K, et al. (2006) Prevalence and incidence of adults

344 consulting for shoulder conditions in UK primary care; patterns of diagnosis and referral.

$345 \quad$ Rheumatology (Oxford) 45:215-221.

346 3. Bergman S, Herrström P, Högström K, et al. (2001) Chronic musculoskeletal pain,

347 prevalence rates, and sociodemographic associations in a Swedish population study. J

$348 \quad$ Rheumatol 28:1369-1377.

349 4. Bamji AN, Erhardt CC, Price TR, Williams PL (1996) The painful shoulder: can

$350 \quad$ consultants agree? Br J Rheumatol 35:1172-1174.

351 5. de Winter AF, Jans MP, Scholten RJ, et al. (1999) Diagnostic classification of shoulder

352 disorders: interobserver agreement and determinants of disagreement. Ann Rheum Dis

$353 \quad 58: 272-277$.

354 6. Liesdek C, Van Der Windt DAWM, Koes BW, Bouter LM (1997) Soft-tissue disorders

355 of the shoulder. A study of inter-observer agreement between general practitioners and

356 physiotherapists and an overview of physiotherapeutic treatment. Physiotherapy 83:12-

$357 \quad 17$.

358 7. Schellingerhout JM, Verhagen AP, Thomas S, Koes BW (2008) Lack of uniformity in 359 diagnostic labeling of shoulder pain: time for a different approach. Man Ther 13:478-483. 
360 8. Michener LA, Walsworth MK, Burnet EN (2004) Effectiveness of rehabilitation for patients with subacromial impingement syndrome: a systematic review. J Hand Ther $17: 152-164$.

9. van der Windt DA, Koes BW, de Jong BA, Bouter LM (1995) Shoulder disorders in general practice: incidence, patient characteristics, and management. Ann Rheum Dis 54:959-964.

10. Gebremariam L, Hay EM, van der Sande R, et al. (2014) Subacromial impingement syndrome--effectiveness of physiotherapy and manual therapy. Br J Sports Med 48:12021208.

11. Brox JI, Gjengedal E, Uppheim G, et al. (1999) Arthroscopic surgery versus supervised exercises in patients with rotator cuff disease (stage II impingement syndrome): a prospective, randomized, controlled study in 125 patients with a 2 1/2-year follow-up. J

373 12. Brox JI, Staff PH, Ljunggren AE, Brevik JI (1993) Arthroscopic surgery compared with supervised exercises in patients with rotator cuff disease (stage II impingement syndrome). BMJ 307:899-903.

376 13. Ketola S, Lehtinen J, Rousi T, et al. (2013) No evidence of long-term benefits of arthroscopicacromioplasty in the treatment of shoulder impingement syndrome: Five-year results of a randomised controlled trial. Bone \& joint research 2:132-139.

379 14. Kukkonen J, Joukainen A, Lehtinen J, et al. (2014) Treatment of non-traumatic rotator cuff tears: A randomised controlled trial with one-year clinical results. The bone $\&$ joint journal 96-B:75-81. 
382 15. Haahr JP, Ostergaard S, Dalsgaard J, et al. (2005) Exercises versus arthroscopic

383 decompression in patients with subacromial impingement: a randomised, controlled study

$384 \quad$ in 90 cases with a one year follow up. Ann Rheum Dis 64:760-764.

385 16. Holmgren T, Bjornsson Hallgren H, Oberg B, et al. (2012) Effect of specific exercise 386 strategy on need for surgery in patients with subacromial impingement syndrome:

387 randomised controlled study. BMJ 344:e787.

388 17. Dickens VA, Williams JL, Bhamra MS (2005) Role of physiotherapy in the treatment of 389 subacromial impingement syndrome: a prospective study. Physiotherapy 91:159-164.

390 18. Itoi E (2013) Rotator cuff tear: physical examination and conservative treatment. J

$391 \quad$ Orthop Sci 18:197-204.

392 19. Kuhn JE, Dunn WR, Sanders R, et al. (2013) Effectiveness of physical therapy in treating 393 atraumatic full-thickness rotator cuff tears: a multicenter prospective cohort study. J $394 \quad$ Shoulder Elbow Surg 22:1371-1379.

395 20. Ginn KA, Herbert RD, Khouw W, Lee R (1997) A randomized, controlled clinical trial of 396 a treatment for shoulder pain. Phys Ther 77:802-809; discussion 810-801.

397 21. Kuhn JE (2009) Exercise in the treatment of rotator cuff impingement: a systematic 398 review and a synthesized evidence-based rehabilitation protocol. J Shoulder Elbow Surg $399 \quad 18: 138-160$.

400 22. Sher JS, Uribe JW, Posada A, et al. (1995) Abnormal findings on magnetic resonance 401 images of asymptomatic shoulders. J Bone Joint Surg Am 77:10-15.

402 23. Milgrom C, Schaffler M, Gilbert S, van Holsbeeck M (1995) Rotator-cuff changes in 403 asymptomatic adults. The effect of age, hand dominance and gender. J Bone Joint Surg 404 Br 77:296-298. 
405 24. Girish G, Lobo LG, Jacobson JA, et al. (2011) Ultrasound of the shoulder: asymptomatic 406 findings in men. AJR Am J Roentgenol 197:W713-719.

407 25. Group MS, Unruh KP, Kuhn JE, et al. (2014) The duration of symptoms does not correlate with rotator cuff tear severity or other patient-related features: a cross-sectional study of patients with atraumatic, full-thickness rotator cuff tears. J Shoulder Elbow Surg

411 26. Krief OP, Huguet D (2006) Shoulder pain and disability: comparison with MR findings. AJR Am J Roentgenol 186:1234-1239.

413 27. Trost SG, Owen N, Bauman AE, et al. (2002) Correlates of adults' participation in physical activity: review and update. Med Sci Sports Exerc 34:1996-2001.

415 28. Escolar-Reina P, Medina-Mirapeix F, Gascon-Canovas JJ, et al. (2010) How do careprovider and home exercise program characteristics affect patient adherence in chronic neck and back pain: a qualitative study. BMC health services research 10:60.

418 29. Rabin A, Irrgang JJ, Fitzgerald GK, Eubanks A (2006) The intertester reliability of the 419 Scapular Assistance Test. J Orthop Sports Phys Ther 36:653-660.

30. Tate AR, McClure PW, Kareha S, Irwin D (2008) Effect of the Scapula Reposition Test on shoulder impingement symptoms and elevation strength in overhead athletes. J Orthop Sports Phys Ther 38:4-11.

423 31. Kibler WB, Sciascia A, Dome D (2006) Evaluation of apparent and absolute supraspinatus strength in patients with shoulder injury using the scapular retraction test. Am J Sports Med 34:1643-1647.

426 32. Lewis JS (2009) Rotator cuff tendinopathy/subacromial impingement syndrome: is it 427 time for a new method of assessment? Br J Sports Med 43:259-264. 
Consensus for physiotherapy for shoulder pain

428

$429 \quad$ Figure and Table legends

430

431 Figure 1: Assessment and Treatment Algorithm for a Patient with Shoulder Pain

432

433 Footnotes:

434 1. Muscle performance deficits may take the form of strength, strength ratio, active or

435

436

437

438 passive length or recruitment pattern deficits

2. Examples of methods to assess symptom reduction with alterations in movement: scapular assistance test [29, 30]; scapular retraction test [30,31]; change of posture [32] 


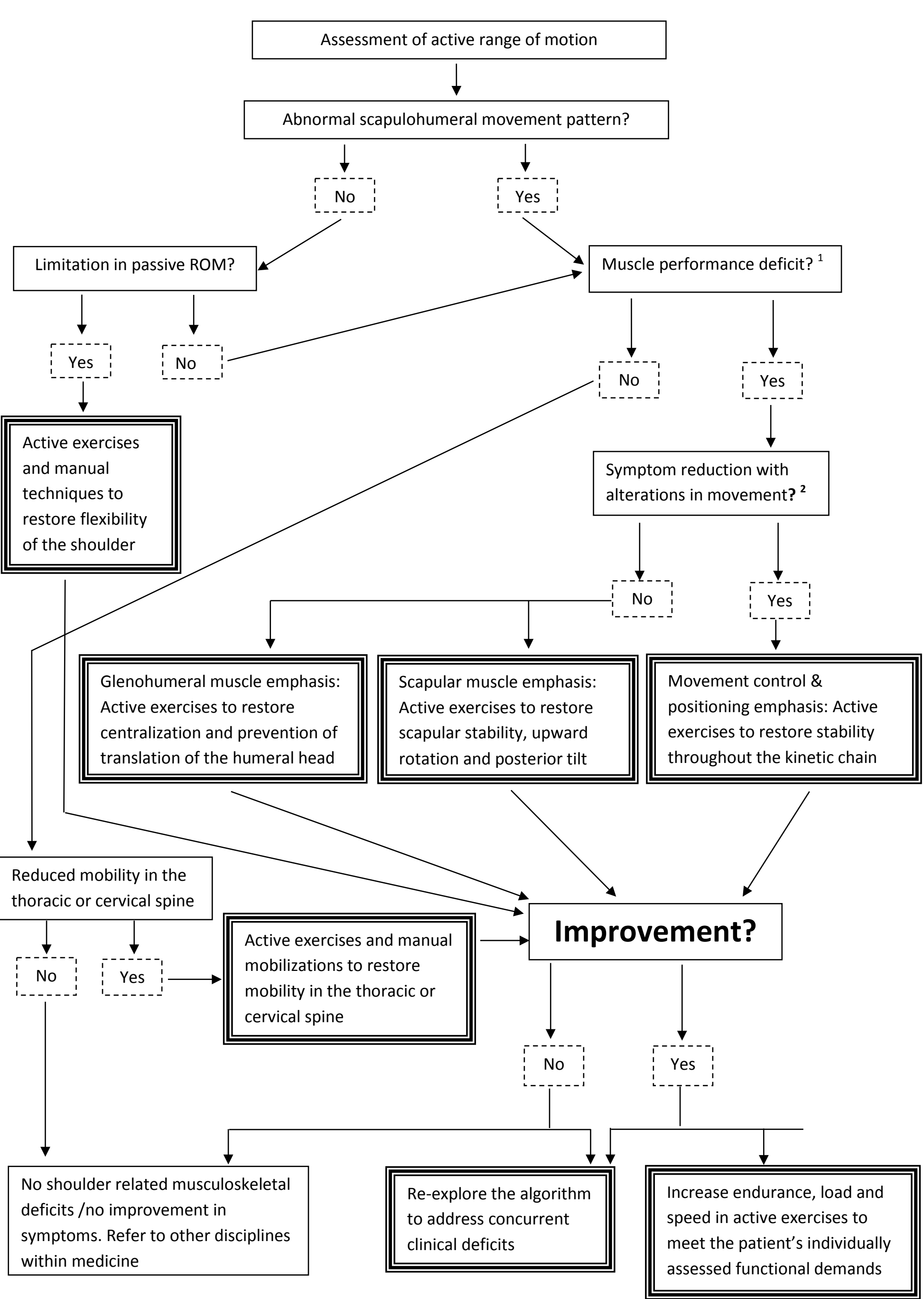

